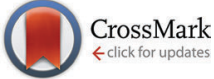

Cite this: Phys. Chem. Chem. Phys., 2016, 18, 18678

Received 30th May 2016,

Accepted 23rd June 2016

DOI: $10.1039 / c 6 c p 04377 a$

www.rsc.org/pccp

\section{Molecular design of light-harvesting photosensitizers: effect of varied linker conjugation on interfacial electron transfer $\dagger$}

\author{
Jianbing Jiang, $\ddagger$ John R. Swierk, $\ddagger$ Svante Hedström, Adam J. Matula, \\ Robert H. Crabtree, ${ }^{*}$ Victor S. Batista, ${ }^{*}$ Charles A. Schmuttenmaer* and \\ Gary W. Brudvig*
}

\begin{abstract}
Interfacial electron transfer dynamics of a series of photosensitizers bound to $\mathrm{TiO}_{2}$ via linkers of varying conjugation strength are explored by spectroscopic and computational techniques. Injection and recombination depend on the extent of conjugation in the linker, where the LUMO delocalization determines the injection dynamics but both the HOMO and HOMO-1 are involved in recombination.
\end{abstract}

The efficient capture and conversion of solar energy into a usable form is a significant research target. Dye-sensitized systems, such as dye-sensitized solar cells (DSSCs) and water-splitting dyesensitized photoelectrochemical cells (WS-DSPECs), utilize high surface area metal oxide scaffolds sensitized with a molecular light-absorbing dye to harvest sunlight and convert it into an electrical potential via an electron-transfer event between the sensitizer and a metal oxide. A solution-phase redox shuttle quenches the sensitizer radical in DSSCs, while in WS-DSPECs it is quenched by a water oxidation catalyst. ${ }^{1,2}$ Unfortunately, in WS-DSPECs and p-type DSSCs, rapid back electron transfer (recombination) significantly limits the power conversion efficiencies that can be attained.,

Molecular design offers a promising strategy for tuning the interfacial electron-transfer kinetics in dye-sensitized energyconversion systems. In DSSCs, a substantial research effort has explored systems of donor-acceptor dyes where the donoracceptor pair is bridged by a $\pi$-conjugated system and has led to significant improvements in power-conversion efficiencies. ${ }^{5-7}$ Alternatively, positioning saturated alkyl linkers between chromophores and anchoring groups is effective at retarding detrimental recombination. ${ }^{8}$ The intermediate case, where the chromophore

Department of Chemistry and Energy Sciences Institute, Yale University, New Haven, Connecticut, 06520, USA. E-mail: robert.crabtree@yale.edu, victor.batista@yale.edu,charles.schmuttenmaer@yale.edu, gary.brudvig@yale.edu $\dagger$ Electronic supplementary information (ESI) available: Full experimental and computational details, including synthetic route, DFT calculations, electron dynamics calculations, Marcus recombination calculations, etc. See DOI: 10.1039/c6cp04377a \$ Equal contributions. and anchor are connected by a partially conjugated bridge, is largely unexplored.

Recently, we demonstrated a phenyl-amide structure that functions as a molecular rectifier based on the spatial asymmetry of the amide moiety. ${ }^{9}$ The amide partially breaks the conjugation within the molecule and depending on its orientation, moves the LUMO closer or further from the Fermi level of the system, making it sensitive to the bias potential and thereby imparting rectifying character.

Inspired by these rectifiers, we developed a novel series of dyes (Ru1-Ru3, Chart 1), as a platform for exploring partially conjugated linking strategies using spectroscopic techniques to characterize the interfacial electron-transfer dynamics in conjunction with quantum chemistry calculations. The dyes, based on the well studied ruthenium tris(bipyridine) motif, were prepared by a standard synthetic strategy, starting with the preparation of the functionalized bipyridine intermediates bearing an ethyl phosphonate group and incorporating amide or ethylene units. The amide bonds were then formed by coupling an amine unit to the carboxylic acid, pre-activated with thionyl chloride for Amide1 or carbodiimide for Amide2. The alkene was formed by condensation of a phosphonate and aldehyde in a Horner-Wadsworth-Emmons reaction. In the next step, the ruthenium tris(bipyridine) complex was synthesized and subjected to chloride-to- $\mathrm{PF}_{6}{ }^{-}$anion exchange for better solubility. Finally, removal of the ethyl groups unveiled the phosphonate anchoring groups. Each target compound was prepared in

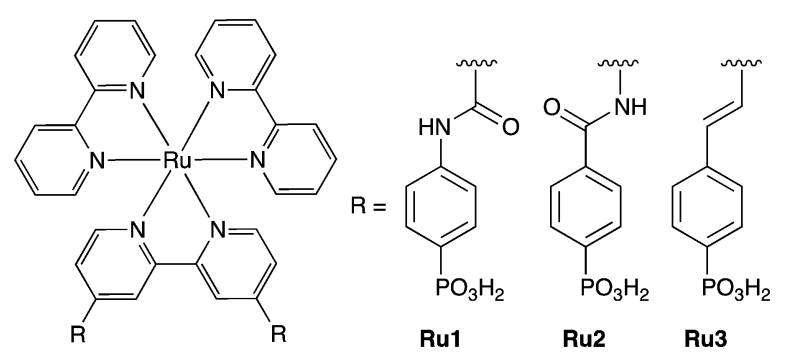

Chart 1 Light-harvesting sensitizers explored in this study. 
modest to high yields, and fully characterized prior to use. Phosphonate groups were used to anchor the chromophores to $\mathrm{TiO}_{2}$ because of their high stability in aqueous environments. ${ }^{10}$

Fig. S1 (ESI $\dagger$ ) shows the steady-state absorption spectra of Ru1-ester, Ru2-ester, and Ru3-ester in acetonitrile. As is typical of ruthenium tris(bipyridine) derivatives, the only absorption feature in the visible region is a strong metal-to-ligand chargetransfer band. For Ru3-ester, the absorption is notably stronger and slightly broader, attributed to the stronger delocalization across the linker. The steady-state emission spectra for the three ester compounds show broad emissions at wavelengths longer than $550 \mathrm{~nm}$. For this class of compounds, the heavy Ru atom induces spin-orbit coupling, facilitating rapid and nearly quantitative intersystem crossing of the excited singlet $\left(\mathrm{S}_{1}\right)$ to the triplet $\left(T_{1}\right)$, with subsequent emission from the $T_{1}$ state. By fitting the emission spectra to a single-mode Franck-Condon function, ${ }^{11,12}$ the energy gap $\left(E_{0-0}\right)$ between the zeroth vibrational levels of the $S_{0}$ and $T_{1}$ states can be determined, see Fig. 1 and Table S1 (ESI $\dagger)$. The $E_{0-0}$ values are $\operatorname{similar}(\sim 2 \mathrm{eV})$ for the three compounds. Table S1 (ESI $\dagger$ ) also shows the vertical transition energies for $S_{0} \rightarrow S_{1}$ calculated with linear-response timedependent DFT as well as the energy difference between the $T_{1}$ and $\mathrm{S}_{0}$ states after optimization with unrestricted DFT (UDFT). Details of the calculations are found in the ESI. $\dagger$ In general, the agreement between the experimental and calculated $E_{0-0}\left(\mathrm{~S}_{0} \rightarrow \mathrm{T}_{1}\right)$ values is very good, differing by less than $0.1 \mathrm{eV}$ in all cases. While $S_{1} \rightarrow S_{0}$ emission cannot be observed experimentally, hot injection from the $\mathrm{S}_{1}$ state into $\mathrm{TiO}_{2}$ is known to occur for other ruthenium tris(bipyridine) derivatives. ${ }^{13}$ DFT calculations suggest that the unrelaxed $\mathrm{S}_{1}$ state of these compounds lies above the optimized $\mathrm{T}_{1}$ by about $0.5 \mathrm{eV}$.

The ground state potential for oxidation $E_{1 / 2}\left(\mathrm{Ru}^{3+/ 2+}\right)$ was determined from cyclic voltammetry in acetonitrile using the ester versions of Ru1, Ru2, and Ru3 (Fig. S2, ESI $\dagger$ ) and was also calculated with UDFT. For Ru1-ester, we determined a potential of 1.32 V vs. NHE, while Ru2-ester and Ru3-ester gave potentials of $1.06 \mathrm{~V}$ and $1.21 \mathrm{~V} v s$. NHE, respectively (Table S1, ESI $\dagger$ ). The excited state reduction potential $E^{0 \prime}\left(\mathrm{Ru}^{3+/ 2+*}\right)$ can be approximated

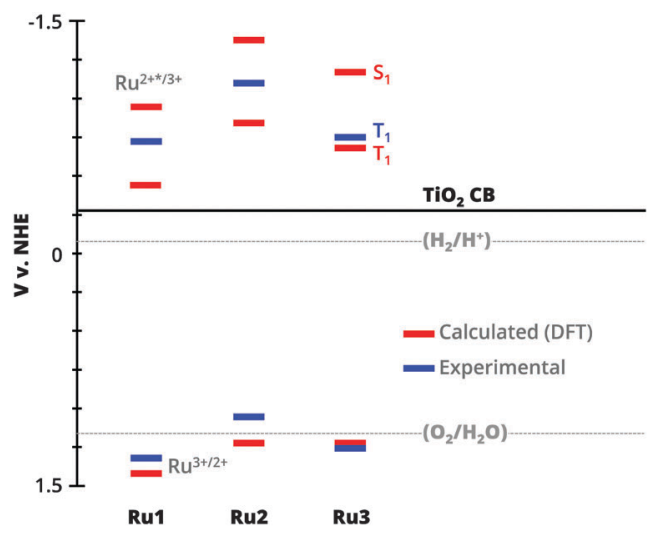

Fig. 1 Experimental and calculated redox potentials for Ru1, Ru2, and Ru3 as well as the position of the anatase $\mathrm{TiO}_{2}$ conduction band and the $\mathrm{H}_{2} / \mathrm{H}^{+}$and $\mathrm{O}_{2} / \mathrm{H}_{2} \mathrm{O}$ couples at $\mathrm{pH} 1$. as the difference between $E_{1 / 2}\left(\mathrm{Ru}^{3+/ 2+}\right)$ and $E_{0-0}$ (Fig. 1). Ru2 exhibits the most negative $E_{1 / 2}\left(\mathrm{Ru}^{3+/ 2+} *\right)$ at $-1.06 \mathrm{~V} v s$. NHE, while Ru1 and Ru3 lie lower in energy at -0.63 and $-0.72 \mathrm{~V} v s$. NHE, respectively. At $\mathrm{pH} 1$, there is significant $\Delta G$ for injection into the conduction band of at least $420 \mathrm{mV}$. The calculated $\mathrm{T}_{1}$ excited-state energies are slightly but consistently underestimated compared to experiments, which correlates with the small underestimations of the reduction potentials. (Fig. S1 and Table S1, ESI + ).

The relative energy levels of the three compounds are rationalized from their chemical structures as follows. The carbonyl group directly attached to the bpy in Ru1 is electronwithdrawing and consequently stabilizes the orbitals in the bpy $\pi$ system. In Ru2, however, the electron-donating amine group raises the bpy $\pi$-orbital energies. This has a larger effect on the LUMOs, which are of pure $\pi^{*}$ character, than on the HOMOs that are mainly Ru-d orbitals but have some bpy- $\pi$ character. The HOMO level directly relates to the ground state potential for oxidation, and the LUMO level relates to the excited state potential since the excitation predominantly corresponds to the promotion of an electron to this orbital. Ru3 exhibits intermediate potentials in all cases due to the very weakly electrondonating vinylene group. The calculated HOMO, LUMO, and HOMO-1 of all dyes are depicted in Fig. 2.

Time-resolved terahertz spectroscopy (TRTS) is a powerful tool for monitoring the injection of an electron into the conduction band of $\mathrm{TiO}_{2} \cdot{ }^{14,15}$ TRTS is an ultrafast, far-infrared technique that is sensitive to the presence of mobile electrons, i.e. electrons in the conduction band. By measuring the decrease in the peak amplitude of the THz pulse, the appearance of electrons in the conduction band can be monitored on the fs-ps timescale. Fig. 3 shows the TRTS scans for Ru1, Ru2, and $\mathbf{R u} 3$ on $\mathrm{TiO}_{2}$ in $0.1 \mathrm{M}$ $\mathrm{HClO}_{4}$. Despite the significant driving force for injection for all three compounds, only Ru2 and Ru3 show substantial injection on a one-nanosecond timescale. Ru1 exhibits less than $20 \%$ of the injection amplitude of Ru3 over the time window permitted by our instrument, despite exhibiting a similar $E^{0 \prime}\left(\mathrm{Ru}^{3+/ 2+*}\right)$ potential. A three-exponential function (described in the ESI $\dagger$ ),

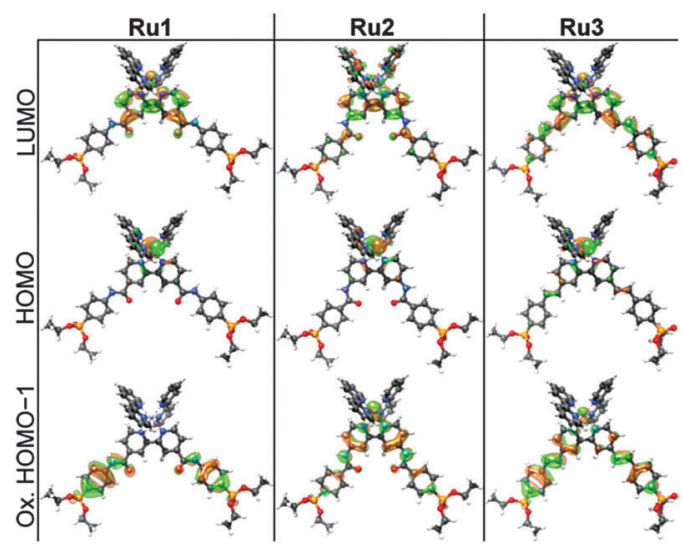

Fig. 2 Ground state HOMO and LUMO of the three esters, along with the HOMO-1 of the structurally relaxed oxidized state. Calculated at the DFT B3LYP/SDD $[R u], 6-311+G(d, p)[P, C, N, H, O] / / S D D[R u], 6-31 G(d)[P, C, N, H, O]$ level of theory. 


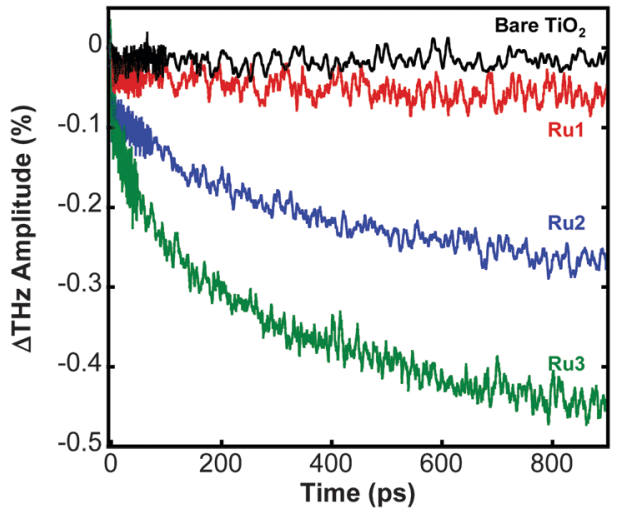

Fig. 3 TRTS scans of Ru1, Ru2, and Ru3 on $\mathrm{TiO}_{2}$ sealed with $0.1 \mathrm{M} \mathrm{HClO}_{4}$. Samples were excited with a $100 \mathrm{~mW}$ Ti:Saphire Laser $(400 \mathrm{~nm}, 35 \mathrm{fs}$, $100 \mathrm{kHz})$

convoluted with a Gaussian instrument-response function of 0.5 fs was fitted to the TRTS scans for Ru2 and Ru3. Because of the small injection amplitude of Ru1, only a biexponential equation was used for this dye.

All three compounds exhibit a component that is instrument response limited ( $<0.5$ ps). Both Ru2 and Ru3 exhibit an intermediate component of 122 ps and 63 ps, respectively, and a slower component of 725 ps and 501 ps, respectively. Ru1 exhibited only an additional slow component of 520 ps. The relative injection amplitudes for $\mathbf{R u} 2$ and $\mathbf{R u} 3$ agree with our previous study of a phosphonated ruthenium tris(bipyridine) on $\mathrm{TiO}_{2},{ }^{16}$ with roughly $20 \%$ of the injection occurring at less than 0.5 ps and about $50 \%$ of the injection occurring at long timescales (Table S2, ESI $\dagger$ ). We suggest that the fast $(<0.5 \mathrm{ps})$ component likely relates to "hot injection" from the $S_{1}$ state, while the long component ( $>500 \mathrm{ps}$ ) corresponds to injection from the vibrationally and structurally relaxed $\mathrm{T}_{1}$ state. The intermediate component may then account for mixed $S_{1}$ and $T_{1}$ injection. Based on this picture, the minimal injection from Ru1 on the $1 \mathrm{~ns}$ timescale suggests that intersystem crossing is much faster than injection from the short-lived $S_{1}$ state and the slow injection $(\sim 6 \mathrm{~ns})$ we observe in the transient absorption experiments (see below) is mostly from $T_{1}$.

The much lower injection yield of Ru1 on a sub-nanosecond timescale is readily rationalized from its LUMO, which is completely localized on the carbonyl and adjacent bpy, far removed from the anchoring phosphonate and thus from the $\mathrm{TiO}_{2}$, see Fig. 2. This localization results from the previously mentioned electron-withdrawing effect of the amide carbonyl group, which stabilizes the bpy-LUMO to the point where it remains unmixed with the remainder of the $\pi$ system. In Ru2 and Ru3, the bpy LUMO is destabilized by the electrondonating amine and vinylene, raising the bpy-LUMO to an energy similar to the remaining part of the conjugated linker, inducing a delocalization towards the anchor. While linker rigidity can inhibit overlap between the chromophore and the anchor group, ${ }^{17}$ that is unlikely to play a role in our case as rotation around the amide and ethylene bridges in Ru1, Ru2, and $\mathbf{R u} 3$ is permitted.
A quantification of how this MO property affects the injection was obtained from electron dynamics simulations, following a previously reported methodology ${ }^{18}$ based on Extended Hückel semi-empirical calculations. The three dyes were anchored to the (101) plane of a 2D-periodic $\mathrm{Ti}_{128} \mathrm{O}_{256}$ slab of anatase via a semi-optimized binding geometry obtained from DFT calculations (Fig. S3, ESI $\dagger$ ). The initial wave packet was created by populating the adsorbate LUMO, the character of which is verified against the DFT-calculated LUMO (Fig. S4, ESI $\dagger$ ). Propagation was calculated by integrating the time-dependent Schrödinger equation (eqn (S4), ESI $\dagger$ ) analytically in time steps of 1.0 fs for 1000 fs. Exponential dampening terms were added to the Ti atoms on the edge and bottom of the slab, reducing the wave packet. The population of the wave packet norm on the adsorbate was then monitored and the remaining fraction of the wave packet tracked to give an estimate of the injection time constant $\tau$, see eqn (S5) (ESI $\dagger$ ) and Fig. 4. The calculated values of $\tau$ obtained for the three dyes: 3724 fs, 1234 fs, and 953 fs for Ru1, Ru2, and Ru3, respectively, are in good agreement with the trend from TRTS. Absolute agreement is not expected due to the approximations in the dynamics calculations, which neglect solvent effects and structural dynamics for example.

Transient absorption spectroscopy (TAS), a time-resolved method for investigating fast (ns-ms) electronic processes, ${ }^{19-22}$ allowed us to monitor the recombination of the injected electron back to the oxidized sensitizer on the microsecond time scale (Fig. 5). The samples were excited with a $2 \mathrm{~mJ}$ pulse at $532 \mathrm{~nm}$ with a beam diameter of $10 \mathrm{~mm}$, and Fig. 5 shows the short $(<3 \mu \mathrm{s})$ and long $(100 \mu \mathrm{s})$ single-wavelength traces monitored at $420 \mathrm{~nm}$. At $420 \mathrm{~nm}$, the loss of absorption corresponds to a bleach of the MLCT band following the formation of $\mathrm{Ru}^{3+}$. While the bleach is instantaneous for Ru2 and Ru3 on the time scale of the measurement, the bleach grows in on the timescale of several nanoseconds for Ru1. A fit of the injection component of the Ru1 TAS gives an injection lifetime of $\sim 6 \mathrm{~ns}$.

A stretched exponential (see ESI $\dagger$ ) was fit to the data from $100 \mathrm{~ns}$ to $100 \mu \mathrm{s}$ to avoid the contribution from the slow ( $\sim 6 \mathrm{~ns}$ ) injection observed for Ru1. For Ru1 the value of $\langle\tau\rangle$

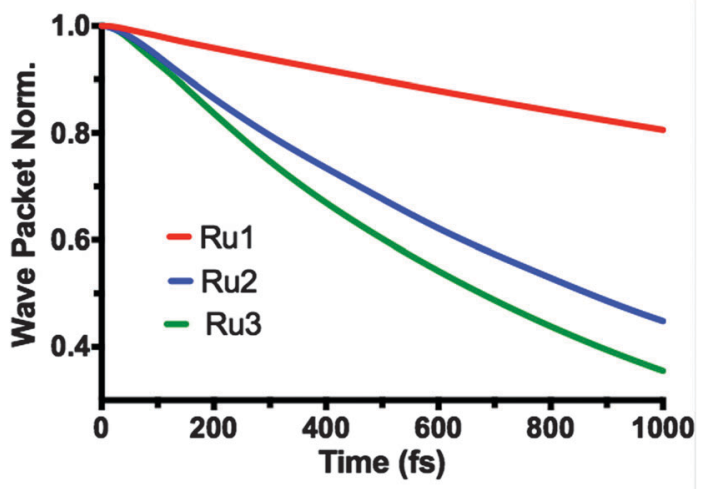

Fig. 4 Extended Hückel dynamics results. The norm of the wave packet plotted in time showing the portion of the original wave packet remaining in the system (i.e. that which has not yet been drawn out of the system by the adsorbing boundary conditions). 


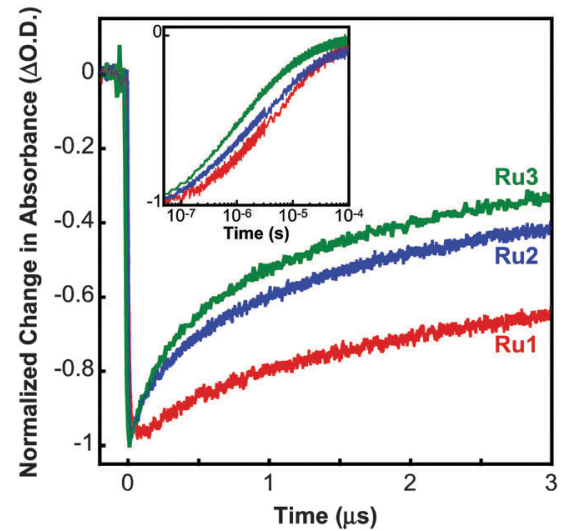

Fig. 5 Transient absorption traces at $420 \mathrm{~nm}$ for Ru1, Ru2, and Ru3 on $\mathrm{TiO}_{2}$ in $0.1 \mathrm{M} \mathrm{HClO}_{4}$. Samples were excited with a $2 \mathrm{~mJ}, 532 \mathrm{~nm}$ pulse $(7 \mathrm{~ns}$, $10 \mathrm{~mm}, 10 \mathrm{~Hz}$ ). Inset: absorbance change on a logarithmic time scale that extends to $100 \mu \mathrm{s}$.

was $23 \pm 4 \mu$ s, while for $\mathbf{R u} 2$ and $\mathbf{R u} 3\langle\tau\rangle$ was $13 \pm 2 \mu$ s and $7 \pm 2 \mu \mathrm{s}$, respectively. The trend in recombination times from electrons in the $\mathrm{TiO}_{2}$ to the three oxidized dyes is rationalized from the calculated extents and energies of the HOMO and HOMO-1 of the oxidized dyes. In all dyes, the HOMO is almost exclusively of $\mathrm{Ru}(4 \mathrm{~d})$ character, localized on the ruthenium core. The spatial separation between this orbital and the $\mathrm{TiO}_{2}$ makes the recombination $\mathrm{TiO}_{2} \rightarrow \mathrm{HOMO}_{\text {dye }}$ dominated by Marcus-type hopping due to very weak coupling (eqn (S6), ESI $\dagger$ ), but this type of electron transfer cannot account for the trend in recombination rates between the dyes. While absent in Ru1, a more efficient pathway for recombination appears in Ru2 and Ru3, where the HOMO-1 of the oxidized species extends across the entirety of the linkers while also exhibiting significant overlap with the HOMO, see Fig. 2. The hole in the oxidized species can easily access the HOMO-1 in Ru2 and Ru3, where a Boltzmann estimate based on the orbital energies suggests that the population ratio of $\mathrm{HOMO}-1 / \mathrm{HOMO}$ is 0.15\%, $1.10 \%$, and $5.73 \%$ at $298 \mathrm{~K}$ for $\mathbf{R u 1}, \mathbf{R u} 2$, and $\mathbf{R u} 3$, respectively. Ru1 consequently has its recombination quenched for two reasons: the HOMO-1 is too deep to be accessible for the hole on the oxidized dye, and it does not overlap spatially with the HOMO.

In order to gauge the interplay of injection and recombination in these sensitizers, we utilized them as sensitizers in DSSCs (Table S3, ESI $\dagger$ ). Of the three sensitizers, Ru2 exhibited the highest short-circuit current, open-circuit voltage, and power conversion efficiency. This suggests that the partial conjugation found in Ru2 provides a better balance of injection and recombination in an actual device than the less conjugated (Ru1) or fully conjugated (Ru3) sensitizers.

To summarize, we have used advanced time-resolved spectroscopic techniques in conjunction with steady-state and timedependent quantum chemistry calculations to follow the electronic dynamics of three new light-harvesting sensitizers in which the energy-level landscape is tuned via small alterations in the chemical structure of the linkers. The linkers impose notable changes in the rates of injection and recombination when anchoring the sensitizers to $\mathrm{TiO}_{2}$ nanoparticles. Dye-sensitized solar cells constructed from these systems show markedly distinct efficiencies. In a broader context, the balance of injection and recombination dynamics being tunable by tailoring the degree of conjugation is likely to be important for many electron-transfer processes beyond dye-sensitized solar cells and water-splitting dye cells.

\section{Acknowledgements}

This work was funded by the U.S. Department of Energy Office of Science, Office of Basic Energy Sciences, under award no. DE-FG02-07ER15909 and by a generous donation from the TomKat Charitable Trust. V. S. B. acknowledges support for the computational work from the Argonne-Northwestern Solar Energy Research (ANSER) Center, an Energy Frontier Research Center funded by the U.S. Department of Energy, Office of Science, Office of Basic Energy Sciences (US DOE-OS-BES) under award no. DE-SC0001059 and the Center for High Performance Computing, Shanghai Jiao Tong University.

\section{Notes and references}

1 J. R. Swierk and T. E. Mallouk, Chem. Soc. Rev., 2013, 42, 2357-2387.

2 K. J. Young, L. A. Martini, R. L. Milot, R. C. Snoeberger III, V. S. Batista, C. A. Schmuttenmaer, R. H. Crabtree and G. W. Brudvig, Coord. Chem. Rev., 2012, 256, 2503-2520.

3 F. Odobel, L. Le Pleux, Y. Pellegrin and E. Blart, Acc. Chem. Res., 2010, 43, 1063-1071.

4 J. R. Swierk, N. S. McCool and T. E. Mallouk, J. Phys. Chem. C, 2015, 119, 13858-13867.

5 Y. Wu and W. Zhu, Chem. Soc. Rev., 2013, 42, 2039-2058.

6 S. Mathew, A. Yella, P. Gao, R. Humphry-Baker, B. F. E. Curchod, N. Ashari-Astani, I. Tavernelli, U. Rothlisberger, M. K. Nazeeruddin and M. Grätzel, Nat. Chem., 2014, 6, 242-247.

7 M. Liang and J. Chen, Chem. Soc. Rev., 2013, 42, 3453-3488.

8 C. Zafer, M. Kus, G. Turkmen, H. Dincalp, S. Demic, B. Kuban, Y. Teoman and S. Icli, Sol. Energy Mater. Sol. Cells, 2007, 91, 427-431.

9 W. Ding, C. F. A. Negre, J. L. Palma, A. C. Durrell, L. J. Allen, K. J. Young, R. L. Milot, C. A. Schmuttenmaer, G. W. Brudvig, R. H. Crabtree and V. S. Batista, ChemPhysChem, 2014, 15, 1138-1147.

10 K. Hanson, M. K. Brennaman, H. Luo, C. R. K. Glasson, J. J. Concepcion, W. Song and T. J. Meyer, ACS Appl. Mater. Interfaces, 2012, 4, 1462-1469.

11 K. Hanson, M. K. Brennaman, A. Ito, H. Luo, W. Song, K. A. Parker, R. Ghosh, M. R. Norris, C. R. K. Glasson, J. J. Concepcion, R. Lopez and T. J. Meyer, J. Phys. Chem. C, 2012, 116, 14837-14847.

12 H. Park, E. Bae, J.-J. Lee, J. Park and W. Choi, J. Phys. Chem. $B, 2006,110,8740-8749$.

13 J. B. Asbury, Y. Q. Wang, E. Hao, H. N. Ghosh and T. Lian, Res. Chem. Intermed., 2001, 27, 393-406. 
14 M. C. Beard, G. M. Turner and C. A. Schmuttenmaer, Phys. Rev. B: Condens. Matter Mater. Phys., 2000, 62, 15764-15777.

15 G. M. Turner, M. C. Beard and C. A. Schmuttenmaer, J. Phys. Chem. B, 2002, 106, 11716-11719.

16 J. R. Swierk, N. S. McCool, C. T. Nemes, T. E. Mallouk and C. A. Schmuttenmaer, J. Phys. Chem. C, 2016, 120, 5940-5948.

17 Z. Yao, L. Ying, C. Yan, M. Zhang, N. Cai, X. Dong and P. Weng, J. Phys. Chem. C, 2014, 118, 2977-2986.

18 L. G. C. Rego and V. S. Batista, J. Am. Chem. Soc., 2003, 125, 7989-7997.
19 S. A. Haque, Y. Tachibana, R. L. Willis, J. E. Moser, M. Grätzel, D. R. Klug and J. R. Durrant, J. Phys. Chem. B, 2000, 104, 538-547.

20 F. Ma, M. Jarenmark, S. Hedström, P. Persson, E. Nordlander and A. Yartsev, RSC Adv., 2016, 6, 20507-20515.

21 M. K. Brennaman, A. O. T. Patrocinio, W. Song, J. W. Jurss, J. J. Concepcion, P. G. Hoertz, M. C. Traub, N. Y. M. Iha and T. J. Meyer, ChemSusChem, 2011, 4, 216-227.

22 P. G. Johansson, Y. Zhang, M. Abrahamsson, G. J. Meyer and E. Galoppini, Chem. Commun., 2011, 47, 6410-6412. 